\title{
Spectroimmunohistochemistry: A Novel Form of MALDI Mass Spectrometry Imaging Coupled to Immunohistochemistry for Tracking Antibodies
}

\author{
Rémi Longuespée, ${ }^{1,2}$ Charlotte Boyon, ${ }^{1,3}$ Annie Desmons, ${ }^{1}$ Olivier Kerdraon,, ${ }^{1,4}$ Eric Leblanc, ${ }^{1,5}$ \\ Isabelle Farré, ${ }^{1,5}$ Denis Vinatier, ${ }^{1,3}$ Robert Day, ${ }^{4}$ Isabelle Fournier, and Michel Salzet ${ }^{1}$
}

\begin{abstract}
MALDI mass spectrometry imaging (MALDI-MSI) is currently used for clinical applications, such as biomarker identification, particularly for the study of solid tumors. The ability to map specific compounds that have been determined to be biomarkers and therapeutic targets is relevant for the evaluation of the efficacy of targeted therapies. This article describes a new method called Spectro-ImmunoHistoChemistry (SIHC), which combines the use of specific antibodies against markers and mass spectrometric imaging in the MS/MS mode. SIHC is based on direct primary antibody-antigen recognition, trypsin digestion of the antibody overlaying the markers of interest in the tissue section, and MALDI-MSI of the tryptic peptides generated from the antibody. This approach has both clinical and pharmacological applications. First, it can be used as a cross-validation method to monitor the presence specifically of a marker in a tissue section. Second, SIHC could potentially be used as a novel technology for tracking specific antibodies after in vivo injection for anti-cancer treatments. Additionally, SIHC could enable novel clinical applications of MSI, such as monitoring the efficacy of cytotoxic antibody treatments.
\end{abstract}

\section{Introduction}

$S_{\mathrm{d}}^{\mathrm{n}}$ NCE THE CONCEPT OF MAPPING MOLECULAR COMPOUNDS directly on tissues using MALDI ion sources was proposed at the end of the 1990s (Caprioli et al., 1997), MALDI mass spectrometry imaging (MALDI MSI) has been used for a wide array of clinical applications (Chaurand et al., 2004; Cornett et al., 2006; Fournier et al., 2003, 2008; Franck et al., 2009b; Lemaire, 2005b; McDonnell et al., 2010; Schwamborn et al., 2007; Seeley and Caprioli, 2008; Stauber et al., 2006). A decade of instrumentation and sample preparation development has led to the optimal detection and spatial resolution for mapping compounds in tissue sections (Angel and Caprioli, 2013; Gagnon et al., 2012). These developments, combined with the use of the appropriate computational tools for data processing, have led to the discovery of biomarkers for diverse pathologies, including cancer (Bonnel et al., 2011;
Djidja et al., 2009; McCombie et al., 2005; Stauber et al., 2008; Trede et al., 2012). MALDI MSI technology provides access to the molecular profiles of benign and malignant sections of solid tumors (Aichler et al., 2013; Balluff et al., 2010, 2011; El Ayed et al., 2010; Elsner et al., 2012; Flatley et al., 2013; Franck et al., 2009b, 2010; Gustafsson et al., 2012; Jones et al., 2013; Lemaire et al., 2005b, 2007a; Longuespee et al., 2012a, 2012b; McDonnell et al., 2010; Meding et al., 2012a, 2012b; Meding and Walch, 2013; Ray-Coquard et al., 2012; Wisztorski et al., 2013). The application of this method is particularly useful for identifying biomarkers in biopsies from early stages of cancer, when the disease is still treatable and the 5-year survival rate of managed patients remains high (Longuespée et al., 2012a, 2012b; Lowe et al., 2013; Meding et al., 2013).

MALDI MSI technology allows for the assessment of hundreds of biomolecular compounds from different molecular classes, and it would be greatly beneficial to be able to

\footnotetext{
${ }^{1}$ Laboratoire PRISM : Protéomique, Réponse Inflammatoire, Spectrométrie de Masse, Cité Scientifique, Villeneuve D’Ascq, Lille Cedex, France.

${ }^{2}$ Institut de Pharmacologie de Sherbrooke, Département de Chirurgie/Urologie, Université de Sherbrooke, Sherbrooke, Québec, Canada.

${ }^{3}$ Hôpital Jeanne de Flandre, Service de Chirurgie Gynécologique, Lille Cedex, France.

${ }^{4}$ Laboratoire d'Anatomie et de Cytologie Pathologiques, CHRU Lille, Lille Cedex, France.

${ }^{5}$ Centre Oscar-Lambret, Département de Cancérologie Gynécologique, Lille Cedex, France.
} 
validate the presence of specific molecular markers in the cancerous sections of tissues using this technology instead of classical microscopy devices. Indeed, the MALDI-MSI-based discovery of a biomarker has to be validated by a complementary method, such as immunohistochemistry, which relies on the use of an antibody that specifically targets the marker of interest. By detecting this specific antibody by mass spectrometry, especially by MALDI-MSI, the validation of the presence of newly discovered biomarkers could be conducted in the same laboratory, without the need for microscopy. This would have a high impact on disease monitoring by enabling the evaluation of the presence of the compounds of interest in diseased tissues. In this context, MSI could support the evaluation of targeted therapies in preclinical or clinical trials. Finally, a potential application of this method is the monitoring of antibody delivery of cytotoxic treatments. Currently, it is unknown whether the antibodies used to enhance the recruitment of cytotoxic antibodies are distributed in a tumor section. It is important to determine how deeply the antibody can penetrate the tumor and at what concentration it is present. This method would enable the detection of antibodies through their tryptic peptides after enzymatic digestion in tissues and could be directly applicable to clinical trials.

Several years ago, we developed and patented a method called Tag-Mass for mapping MSI-specific targets (Gagnon et al., 2012; Lemaire et al., 2007a; Stauber et al., 2009). One application of the Tag-Mass technology is MSI based and uses photocleavable, tagged antibody probes grafted to specific antibodies for a given biomarker (Gagnon et al., 2012; Lemaire et al., 2005a, 2007b; Stauber et al., 2009). This strategy relies on the design of specific antibodies that are used for immunohistochemistry (IHC), which are linked to tag peptides of known sequences (Gagnon et al., 2012) and can be detected by mass spectrometry. After IHC preparation, the presence of the linked peptides is mapped on the surface of the tissue, and the identity of each antibody is determined by the MS/MS analysis of these linked peptides. Although this method is highly efficient and reproducible, the design of the linked antibodies remains expensive.

In this work, we present a simple and fast method, called Spectro-ImmunoHistoChemistry (SIHC), which combines IHC and MALDI MSI without requiring the design of specific antibodies to image targeted antigens. In the SIHC method, tryptic digests of the primary antibodies are directly mapped against the tissue marker of interest. This is achieved by performing an on-tissue enzymatic digestion after primary antibody binding to the tissue section. Resulting tryptic peptides are then detected directly from the tissue and followed by imaging. In the present article, the proof-of-concept of the SIHC method is presented using ovarian cancer as a clinical model. Additionally, using MALDI MSI, we have discovered a potential novel marker for this pathology: the C-terminal fragment of the activator of the immunoproteasome PA28 (Reg Alpha) (El Ayed et al., 2010; Lemaire et al., 2005, 2007a; Longuespee et al., 2012a). The Reg Alpha C-terminal fragment is one of the markers of immunosuppressive events that occur during the early phases of disease progression (El Ayed et al., 2010; Lemaire et al., 2005b, 2007a; Longuespée et al., 2012a; 2012b). Using SIHC, we validate the statement that RegAlpha is found at the early stages of all ovarian cancerous lesions, making it a good candidate for the early screening of ovarian cancer development.

\section{Materials and Methods}

\section{Materials}

$\alpha$-Cyano-4-hydroxycinnamic acid (CHCA), trifluoroacetic acid (TFA), aniline, PBS, ammonium bicarbonate, dithiothreitol, iodoacetamide, bovine serum albumin, and triton were obtained from Sigma-Aldrich (Saint-Quentin Fallavier, France). Trypsin was obtained from Promega (Charbonnieres, France). Acetonitrile p.a., methanol p.a., ethanol, and acetone were provided by Biosolve B.V. (Valkensvaard, Netherlands). Polyclonal antibodies against Reg Alpha were purchased from Invitrogen (Illkirch, France). Mounting media for tissues stainings was provided by Vectashield. Histological glass slides were purchased from Thermo Fisher (Illkirch, France).

\section{Samples}

Tissues were obtained with informed consent and institutional review board approval (CCPPRBM Lille: CP 05/83) from patients undergoing any ovarian tumor resection at Hospital Jeanne de Flandre of Centre Hospitalier Universitaire de Lille. Three fresh, frozen tissues were used for this investigation, corresponding to stage I to stage IV endometrioid and serous cancer tissues. Patient information was collected, including gender, age, treatment received before and after the surgery, extent of the surgery, current status (alive, alive with progressive disease, or deceased), cause of death if applicable, and survival time from the time of the original pathological diagnosis. Samples were collected at the time of the surgery, immediately frozen at $-50^{\circ} \mathrm{C}$, in cold isopentane, and stored at $-80^{\circ} \mathrm{C}$ until analysis.

\section{Tissue preparation}

Ten micrometer-thick frozen biopsy sections were cut using a cryostat and thaw-mounted on histological glass slides. Tissues were then dried in a dessicator. The sections were washed for $1 \mathrm{~min}$ in acetone, $15 \mathrm{sec}$ in a solution of $70 \%$ ethanol, $15 \mathrm{sec}$ in a solution of $95 \%$ ethanol and, finally, $1 \mathrm{~min}$ in chloroform (Lemaire et al., 2006).

Hematoxylin eosin safran staining. For hematoxylin eosin safran staining (HES), the sections were heated for $5 \mathrm{~min}$, colored with hematoxylin for $3 \mathrm{~min}$, rinsed with water, bathed twice in a solution of $156 \mathrm{~mL}$ of $95 \% \mathrm{EtOH}, 44 \mathrm{~mL}$ of $\mathrm{H}_{2} \mathrm{O}$, and $80 \mu \mathrm{L}$ of $\mathrm{HCl}(1 \mathrm{~N})$, and then bathed in water. Then, the samples were bathed in $35 \% \mathrm{NH}_{4} \mathrm{OH}$ in $200 \mathrm{~mL} \mathrm{H}_{2} \mathrm{O}$, washed for $5 \mathrm{~min}$ with water, and bathed in $80 \%$ ethanol. Next, the sections were colored with eosin for $10 \mathrm{sec}$ and bathed twice with $95 \%$ ethanol and twice with $100 \%$ ethanol. After staining with safran $(10 \mathrm{~g} / \mathrm{L}$ in $100 \% \mathrm{EtOH})$ for $6 \mathrm{~min}$, the slides were bathed twice in $100 \% \mathrm{EtOH}$ and once in xylene for $1 \mathrm{~min}$. Histopathologic diagnoses were made from these H\&E-stained sections by the pathologist (Olivier Kerdraon) without knowledge of the original clinical diagnosis. Consecutive sections were used for SIHC and the HES staining.

Immunohistochemistry of the Reg-alpha Cter fragment in serous ovarian cancer tissues for the SIHC procedure. The prepared tumor tissue sections were incubated at room temperature with $500 \mu \mathrm{L}$ of buffer (0.1 M PBS, $1 \%$ BSA, $1 \%$ normal goat serum, and $0.05 \%$ Triton $\mathrm{X} 100)$ for $30 \mathrm{~min}$. The same buffer was used to dilute the Reg-alpha antibody solution 
(1:100; Zymed Laboratories, Invitrogen, ref. 38-2400). The tissues were incubated with the primary antibody in the buffer overnight at $4^{\circ} \mathrm{C}$. After incubation, tissue sections were rinsed three times for $10 \mathrm{~min}$ with PBS and twice for $5 \mathrm{~min}$ with HPLC-grade water. The tissues were then rinsed three additional times for $5 \mathrm{~min}$ with ultrapure water to remove salts from the buffer, and the sections were then dried at room temperature before trypsin application.

\section{Molecular mass spectrometry analyses}

Trypsin deposition and digestion. Two modes of trypsin deposition were used for the on-tissue trypsin digestion of antibodies, micro-spraying, and micro-spotting.

For trypsin deposition by micro-spraying, $300 \mu \mathrm{L}$ of a solution of $40 \mu \mathrm{g} / \mathrm{mL}$ of trypsin that was diluted in $50 \mathrm{mM}$ $\mathrm{NH}_{4} \mathrm{HCO}_{3}$ was sprayed using a vibrational micro-sprayer (ImagePrep, Bruker Daltonics, Germany), with 13 cycles of $1.5 \mathrm{sec}$ of spraying, $240 \mathrm{sec}$ of incubation, and $30 \mathrm{sec}$ of drying.

For trypsin deposition by micro-spotting, a piezoelectric automated micro-spotting device (CHIP 1000, Shimadzu, Kyoto, Japan) was used. Digestion was performed using $20 \mu \mathrm{g} / \mathrm{mL}$ trypsin in $50 \mathrm{mM} \mathrm{NH}_{4} \mathrm{HCO}_{3}$ in a final volume of $12.5 \mathrm{~nL}$ (Franck et al., 2009a). The distance between two spots was set at $250 \mu \mathrm{m}$.

Matrix deposition. The matrix deposition mode was chosen according to the trypsin deposition method. CHCA/aniline solid ionic matrix was used for these experiments. CHCA/aniline was prepared with an equivalent molarity of CHCA and aniline in ACN/0.1\% TFA, 7:3, v/v in $\mathrm{H}_{2} \mathrm{O}$. For CHCA/aniline micro-spraying, a specific method of deposition was created. In the first phase, the deposition was initialized with short nebulization and incubation times to spray small droplets of matrix, giving rise to small crystals. Spray and incubation times were then programmed at longer durations in the following steps to spray larger droplets (Bonnel et al., 2011). For microspotting, a total of $20 \mathrm{~nL}$ of $10 \mathrm{mg} / \mathrm{mL}$ $\mathrm{CHCA} /$ aniline was placed on the same spots where trypsin was deposited (Franck et al., 2009a).

On-tissue digestion and direct analysis. The digestion reaction was prolonged for sprayed samples by incubating the tissue sections in incubation chambers previously designed by our team (Longuespée et al.) for $2 \mathrm{~h}$, as the spraying time itself is not long enough for a sufficient digestion reaction. After $2 \mathrm{~h}$, the tissue was immediately dried by desiccation and the matrix was deposited.

After the on-tissue digestion and matrix deposition, direct tissue analysis was performed on the MALDI LTQ Orbitrap XL (Thermo Scientific, Bremen, Germany) to assess the spectral quality resulting from the digestion and matrix deposition efficiencies. Approximately 20 scans per digested point in the FTMS mode in the mass range $600-4000 \mathrm{~m} / \mathrm{z}$ with a nominal mass resolution of 100000 at m/z 400 were acquired.

MALDI imaging. The images were acquired using a MALDI LTQ Orbitrap XL (Thermo Scientific, Bremen, Germany).

Spectra were acquired using the Xcalibur 2.0.7 SP1 software (Thermo Fisher Scientific, Bremen, Germany). The LTQ Orbitrap instrument was operated in the positive ion mode over a high mass range $(\mathrm{m} / \mathrm{z}=600-4000)$. The instrument was equipped with an intermediate pressure (75 mTorr) MALDI source using a nitrogen laser (MNL 100; Lasertechnik Berlin), providing an output at $337 \mathrm{~nm}$ with a maximum energy of $80 \mu \mathrm{J}$ per pulse and a repetition rate of $60 \mathrm{~Hz}$. The Orbitrap analyzer was used for MS data acquisition, with a mass resolving power of 30,000 FWHM. Automatic gain control was turned off, and other data acquisition conditions were as follows: laser power per pulse of $10 \mu \mathrm{J}, 25$ laser pulses per spectra, and two scans averaged per pixel. The image reconstructions were performed using the ImageQuest software. The distance between raster points was set to $250 \mu \mathrm{m}$. Image analysis and data visualization were performed with the Imagequest1.0.1 software (Thermo Fisher Scientific, Bremen, Germany).

\section{Antibody chain separation and identification}

Gel electrophoresis. Heavy and light chains of the anti-Cterminal Reg-alpha polyclonal antibodies were reduced using $10 \mathrm{mM}$ DTT, and cysteine residues were subsequently alkylated using $100 \mu \mathrm{L}$ of iodoacetamide at $15 \mathrm{mM}$. Electrophoresis gels were prepared at $12 \%$ for the separation gel and $4 \%$ for the concentration gel. Migration proceeded for $2 \mathrm{~h}$ at 230 volts. After migration, the gels were stained with Coomassie Blue for $24 \mathrm{~h}$. After staining, the gels were bathed in distilled water for 1 day.

In-gel digestion of antibody heavy and light chains. Before trypsin digestion, the gels were washed in water for 2 days. Bands of interest were cut and rinsed with $100 \mu \mathrm{L}$ of $50 \mathrm{mM}$ ammonium bicarbonate buffer and then with $50 \mathrm{mM}$ ammonium bicarbonate buffer containing water:acetonitrile $2: 8 \mathrm{v} / \mathrm{v}(\mathrm{pH}$ ) to remove the remaining Coomassie Blue and allow shrinking of the gel pieces. Then, the gel fragments were incubated overnight with $50 \mu \mathrm{L}$ of $50 \mathrm{mM}$ trypsin in $50 \mathrm{mM}$ ammonium bicarbonate buffer. After vortexing, the supernatant was retained, and $100 \mu \mathrm{L}$ of $\mathrm{ACN}$ :water $1: 1 \mathrm{v} / \mathrm{v}$ was added to the gel fragments. After vortexing, the resulting supernatant was recovered and mixed with the first supernatant. The solution was then dried in a vacuum concentrator, and the peptides were suspended in $20 \mu \mathrm{L}$ of water $/ 2 \%$ acetonitrile $/ 0.1 \%$ of a solution of $98 \%$ formic acid.

Mass spectrometry of digested peptides from antibody chains. After digestion and matrix deposition, peptide analysis was performed on a MALDI LTQ Orbitrap XL previously used for MS or MS/MS analyses. Approximately 20 scans per digested point in FTMS mode in the range 600-4000 with a nominal mass resolution of 100000 at $\mathrm{m} / \mathrm{z} 400$ were acquired.

\section{Results}

Our group previously developed and patented a method for mapping specific compounds in ovarian cancer tissues (Fournier et al., 2006; Lemaire et al., 2005a, 2007b). In this method, called Tag Mass, an antibody is linked to a lasercleavable peptide of known sequence. The image of the $\mathrm{m} / \mathrm{z}$ ratio of this peptide is generated, and the peptide's sequence is determined by MS/MS (Lemaire et al., 2007b). Here, another method to screen for the presence of antibodies raised against specific markers in tissue sections is described. This technology relies on the direct detection of antibodies on tissues 
through their tryptic cleavage patterns. Only a characterization step is needed before performing SIHC assays. We decided to focus our efforts on developing a method for tracking specific compounds that bind to the C-terminal fragments of Reg-alpha. We previously discovered this compound by MALDI MSI and found that it was specifically present in ovarian cancer biopsies (Lemaire et al., 2007a; Longuespee et al., 2012a).

\section{SpectrolmmunoHistoChemistry procedure}

The workflow of the method is presented in Figure 1. The proposed method relies on a combination of IHC and MSI and is named Spectro-ImmunoHistoChemistry (SIHC). Because native antibodies are difficult to detect due to their high mass, trypsin digestion prior to MALDI detection is recommended. Heavy and light chains can be separated following reduction and alkylation (R\&A) (Fig. 1). The antibody used to test this approach is the polyclonal antibody raised against the Cterminal fragment of the 11S proteasome activator complex, named PA28 or Reg-alpha. We have already validated this marker as being indicative of ovarian cancer tissues with IHC (Longuespée et al., 2012a). Thus, as shown in Figure 1A (see inset SDS-PAGE, lane 2), without R\&A, the anti-C-terminal fragment of Reg-alpha antibodies is detected at $150 \mathrm{kDa}$, with a large smear from 75 to $150 \mathrm{kDa}$ revealing that the proteins are denatured but that the heavy and light chains may remain linked. As shown in Figure 1A, in lanes 3 and 4 of the SDSPAGE gel, the antibody chains are separated after R\&A (light chains at $25 \mathrm{kDa}$ and heavy chains at $50 \mathrm{kDa}$ ). We also observe large smeared regions for the heavy and light chains. The appearance of the smears reveals the large heterogeneity of glycoforms in polyclonal antibodies for heavy and light chains. We digested each chain group with trypsin to generate a general profile of the antibody (Fig. 2A). These experiments were conducted three times. Peptide mass fingerprints from gel electrophoresis band digests were then compared with those obtained after the SIHC procedure on cancerous regions of ovarian tissue (Fig. 2B).

In contrast with classical immunohistochemistry assays, only the primary antibody is required for SIHC because the detection is performed during the MSI analysis. The primary antibody was placed on the tissue section and incubated overnight at $4^{\circ} \mathrm{C}$. The slice was then subjected to a washing procedure before R\&A, followed by an on-tissue trypsin digestion. For SIHC analysis, the step consisting of the incubation with a secondary antibody, as in classical immunohistochemistry, was skipped and a layer of matrix was

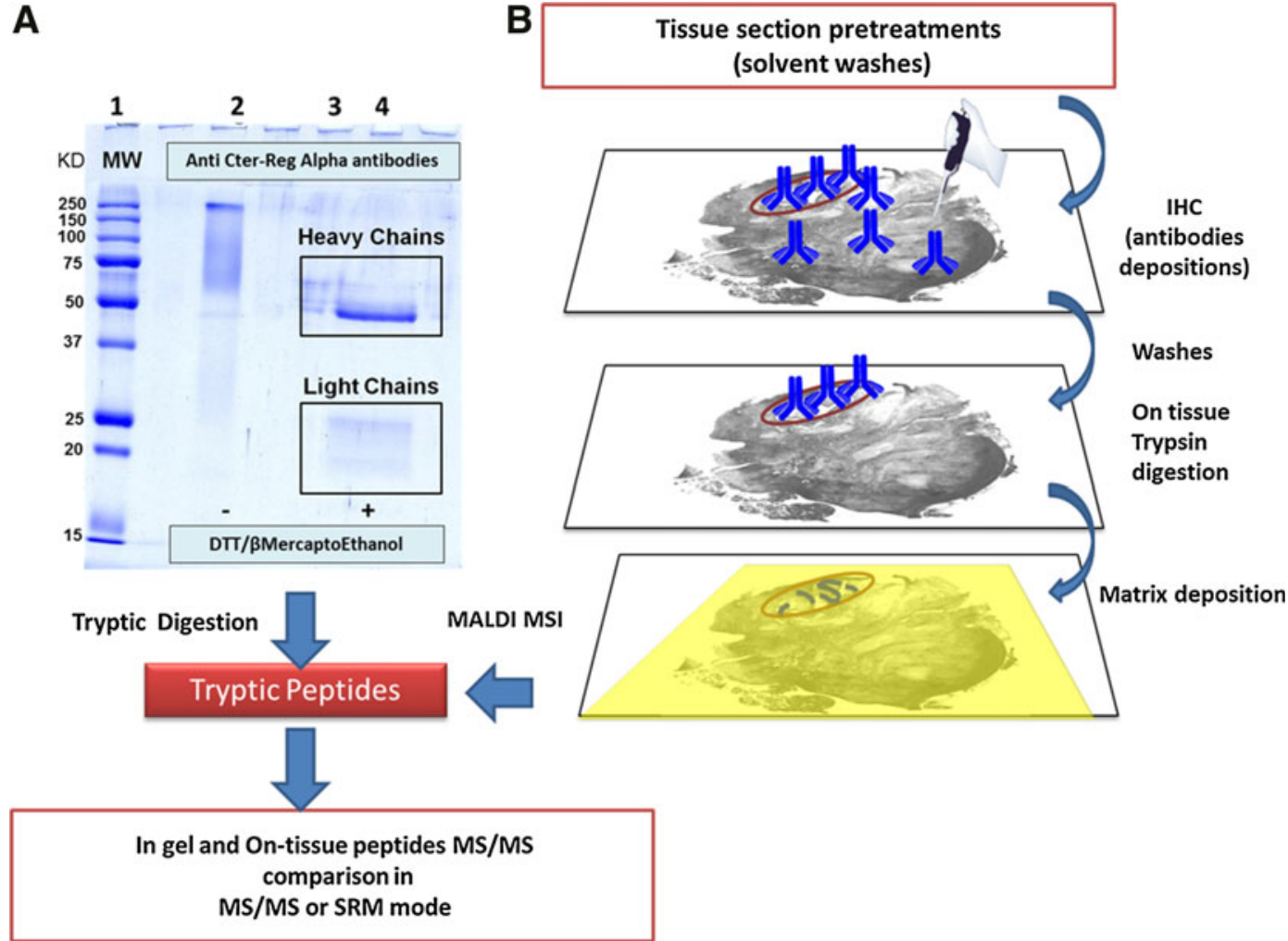

FIG. 1. SIHC workflow. (A) SDS-PAGE gel of the antibody directed against the C-terminal fragment of Reg alpha in the presence or absence of DTT. Separate antibody heavy and light chains were then digested with trypsin before MALDI-MS analysis for specific PMF identification. In parallel, (B) corresponds to a classical incubation of the primary antibody performed on top of the tissue slice. DTT, trypsin, and the matrix were deposited on the tissue in succession before imaging by MALDI MS. The MS/MS profiles of the tryptic peptides shared between the tissue and the in-gel digests were compared, with specific images of the ions corresponding with the digested antibody chains used to determine antibody localization. 

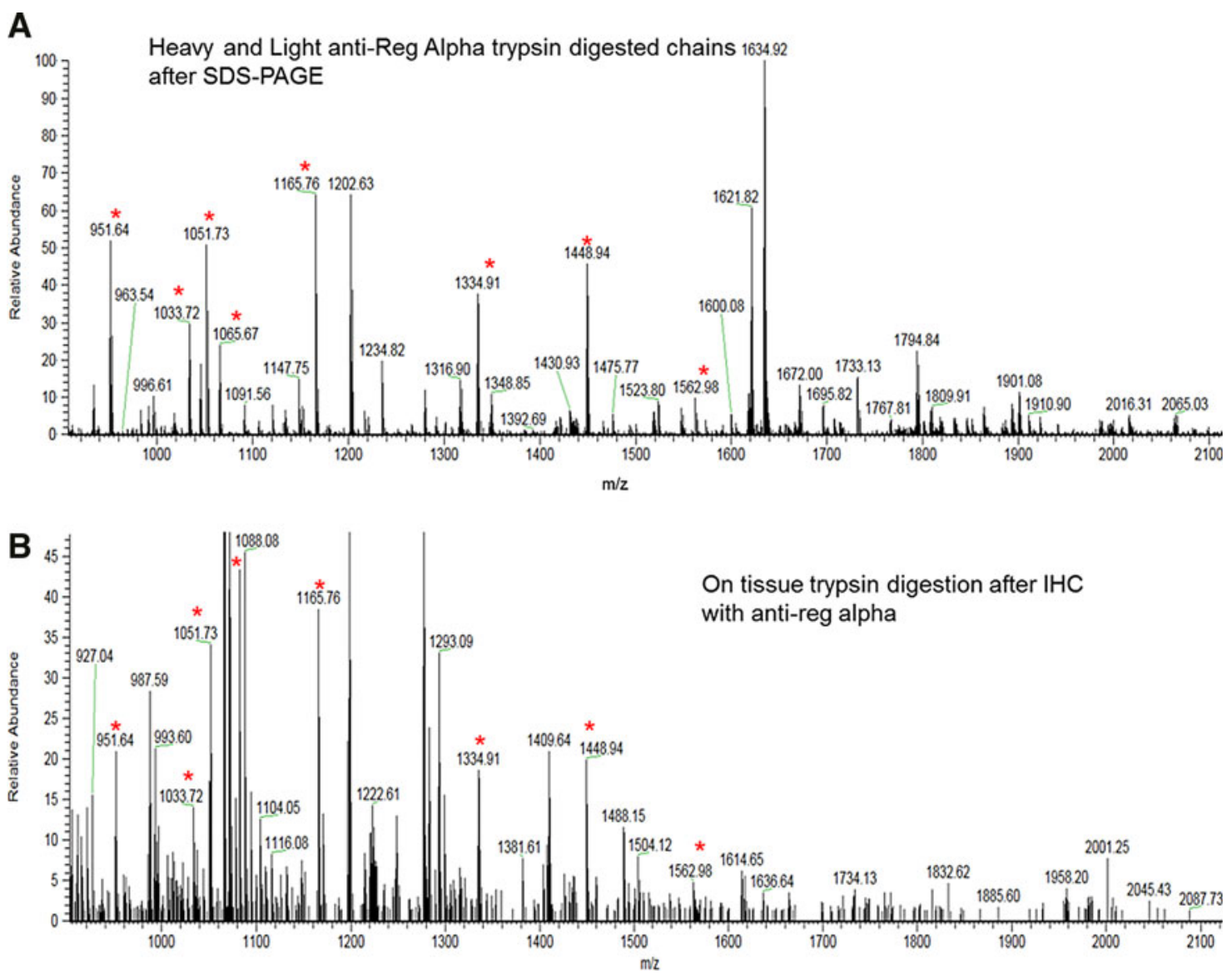

FIG. 2. Comparison of the MALDI MS spectra of heavy and light chains of anti-reg alpha after either electrophoresis gel digestion (A) or on-tissue digestion using a stage I serous ovarian cancer tissue (B). An asterisk $\left(^{*}\right)$ indicates the ions in common. The blue arrow and line indicate the benign zone, and the red arrow and line indicate the cancerous zone.

immediately deposited for the desorption-ionization of the digested fragments and MSI mapping (Fig. 1B). As mentioned above, the principle of this approach is very simple and relies on a direct comparison between the profile of the digested antibody (the peptide mass fingerprint, or PMF) obtained after in-gel trypsin digestion (Fig. 2A) and the PMF of digested peptides found in the tissue after antibody incubation, followed by trypsin digestion (Fig. 2B). For a better comparison, all trypsin-digested fragments were removed from the obtained peaks list, and we focused our attention on a selected window (.e., for ions ranging from $\mathrm{m} / \mathrm{z}$ ratios of 900 to 2100). The antibody recognized the biomarker from the complete protein and the Cter fragment protein. Moreover, these analyses were performed on a MALDI LTQ Orbitrap instrument, allowing for the acquisition of highly resolved mass spectra. In this analysis, we detected several common ions between electrophoresis gels and MALDI images (i.e., ions at $\mathrm{m} / \mathrm{z}$ ratios of $951.62,1033.72,1051.73,1165.76,1334.91,1448.95$, and 1562.98).

\section{SIHC validation in MS mode}

Validation on Stage IV serous ovarian carcinoma samples. To validate the SIHC protocol, we analyzed Stage IV serous ovarian carcinoma (OVC) tissues using a MALDI-Orbitrap instrument. Ions at $\mathrm{m} / \mathrm{z}$ ratios of $1165.76,1334.91$,
1448.95, and 1562.98, corresponding to tryptic peptides of the C-terminal fragment of the Reg-alpha antibody, were tracked through the cancerous zones of the biopsies (Fig. 3A). All specific ions were detected in the tumor region and, to a lesser extent, in the benign region, which agrees with previous work. This result showed that the antibody specifically recognized the biomarker at the level of the epithelial cells in both the nucleus and the cytoplasm in the carcinoma region and only in the nucleus in benign tissue (Lemaire et al., 2007a; Longuespée et al., 2012a). From 376 samples, this marker reflects a prevalence of $77.66 \pm 8.77 \%$ with a correlation coefficient value of $0.601(p>0.001)$ between OVC and benign tissue (Longuespée et al., 2012a). This result confirms the effectiveness of this strategy. This difference in ion detection relates to ion intensity detection in the PMF after gel electrophoresis (Fig. 2A) or on-tissue digestion (Fig. 2B) and can be explained by ionization differences due to the peptide structure and ion suppression. Moreover, some ions are preferentially detected in certain stages and grades of OVC, but in the majority of the cases, the ions at $\mathrm{m} / \mathrm{z}$ ratios of 1165.76 , $1334.91,1448.94$, and 1562.98 are detected.

Validation on early stages of serous ovarian carcinoma samples. The SIHC protocol was then applied to early stage OVC (Stage Ia, Fig. 4 and Stage Ic, Fig. 3B). Patient samples 


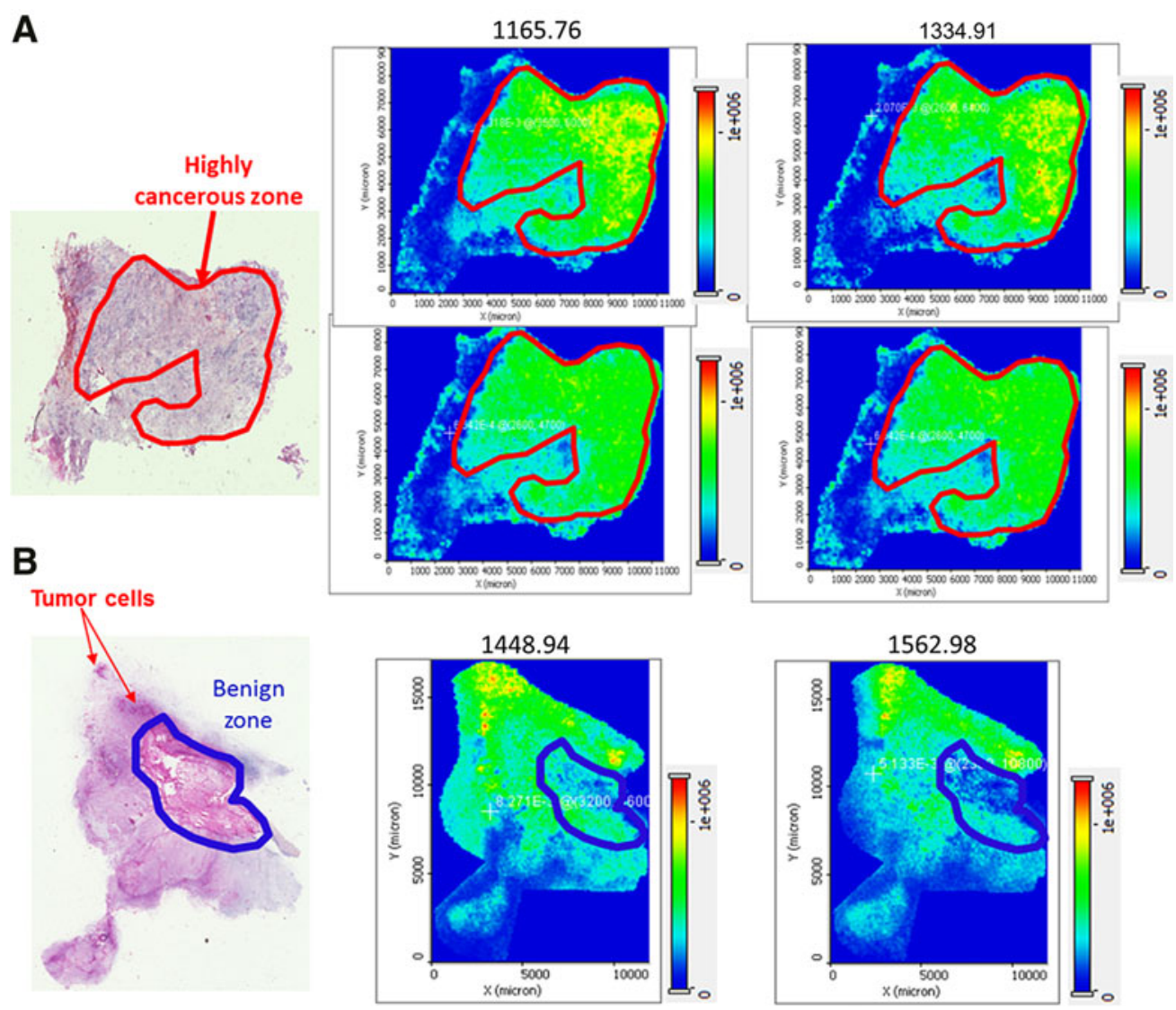

FIG. 3. MALDI molecular images $(250 \mu \mathrm{m})$ of common $\mathrm{m} / \mathrm{z}$ values determined by electrophoresis gel digestion and digestion of Reg Alpha antibody on ovarian carcinoma tissue (red line). Trypsin and the matrix (HCCA/aniline) were sprayed using an automatic sprayer. Specific ions from trypsin-digested antibody chains were detected in the cancerous area. These experiments were performed on a MALDI LTQ Orbitrap. (A) Stage IV OVC. Ions detected have $\mathrm{m} / \mathrm{z}$ ratios of 1165.76, 1334.91, 1448.94, and 1562.98. (B) Stage Ic OVC. Ions detected have $\mathrm{m} / \mathrm{z}$ ratios of 1448.94 and 1562.9. The blue arrow and line indicate the benign zone, and the red arrow and line indicate the cancerous zone.

with stage Ic OVC had both cancerous cells and a benign region (Fig. 3B). Ions at $\mathrm{m} / \mathrm{z}$ ratios of 1448.94 and 1562.98 were detected solely in cancerous cells and not in the benign region (Fig. 3B). A margin region from endometrioid stage Ia OVC [i.e., a small region covering both benign and carcinoma tissue, was selected (Fig. 4B)]. After microspotting for the deposition of trypsin and the ionic solid matrix (HCCA/aniline), the tissue was analyzed in this margin region to compare the cancerous and benign zones in a more restricted window (Fig. 4A). Under these conditions, some specific ions corresponding to digested peptides of the heavy or light antibody chains (ions at $\mathrm{m} / \mathrm{z}$ ratios of 951.64, 1051.73, 1165.76, 1448.94, 1523.8, and 1562.98) were imaged (Fig. 4C). These ions were detected in the cancerous portion but not in the healthy portion of the tissue section, confirming the specificity and selectivity of the SIHC technique (Fig. 4C).

\section{Validation of SIHC in MS/MS mode}

To support the data observed in the MS mode, images were then obtained by tracking the signatures of ions obtained in the MS/MS mode. Selected daughter ions can be mapped using this method, and known peptides of the antibody can be automatically detected and mapped based on the MS/MS signature. Figure 5 presents the molecular images based on the fragments of the ion at an $\mathrm{m} / \mathrm{z}$ value of 1523.79 detected in the tumor region of the margin (Fig. 4C). MSI images of ions were observed at $\mathrm{m} / \mathrm{z}$ ratios of 786.08, 890.4, 1147.01, 1261.6, and 1523.79. All of these daughter ions were present in the tumor region but not in the benign part of the tissue section. These data establish that the SIHC technique can be used in both the MS and MS/MS modes.

\section{Discussion}

These experiments demonstrated that IHC and MALDI MSI methods can be combined to enable powerful detection and mapping of antibodies in biological samples. Specific MALDI MSI of an antibody can now be performed in an inexpensive assay that is not time intensive. We propose a method for the cross-validation of the presence of marker compounds in tissues, using MALDI MSI as the method to detect primary antibodies. This allows for the direct confirmation of molecular localization without the use of secondary antibodies and microscopes, as in this approach, the mass spectrometer acts as a microscope. This opens new 
A
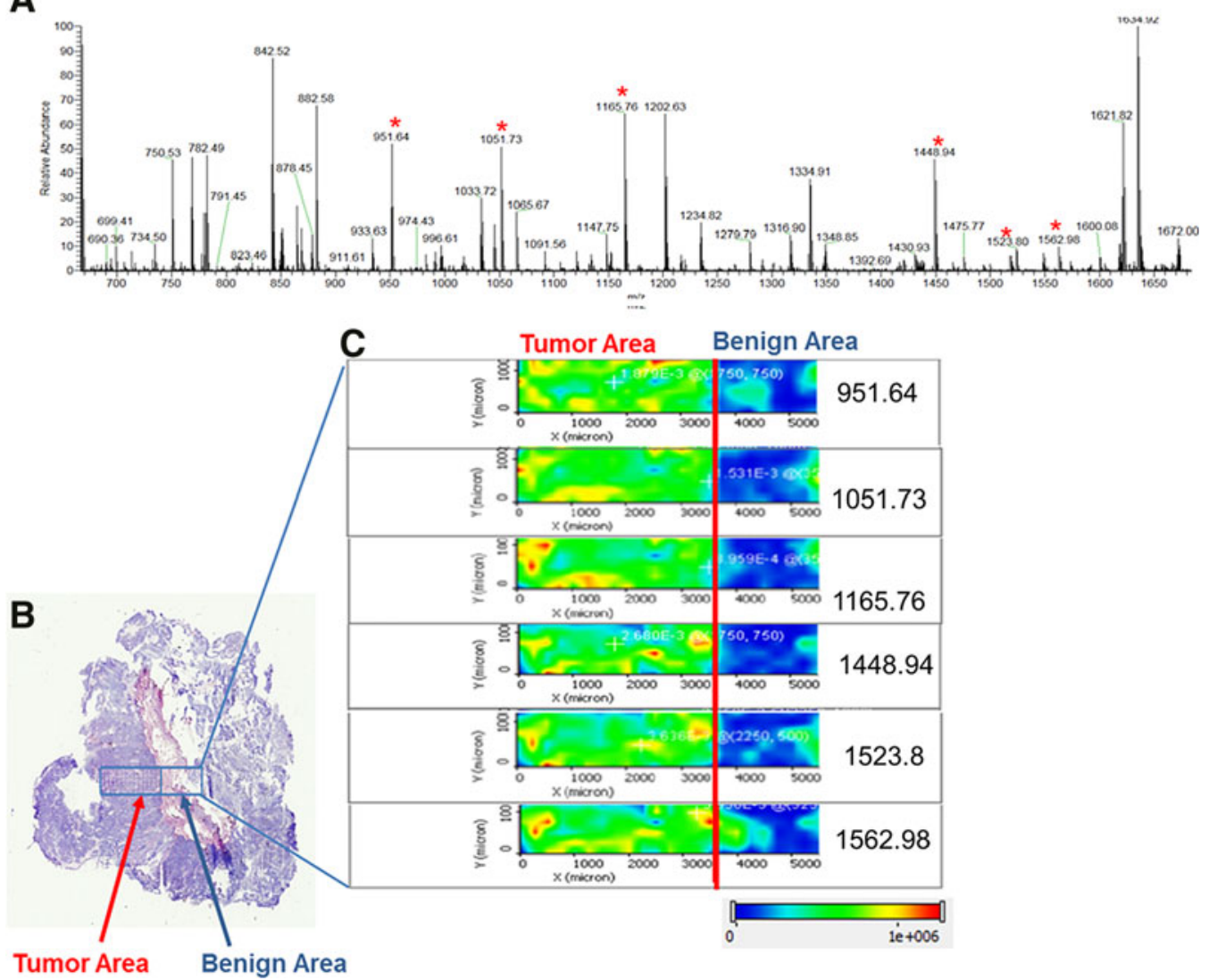

FIG. 4. MALDI molecular images $(250 \mu \mathrm{m})$ of common $\mathrm{m} / \mathrm{z}$ values found by electrophoresis gel digestion and digestion on stage Ia endometrioid OVC tissue at the margin between the cancerous and benign tissue (red line). Trypsin and matrix (HCCA/aniline) were spotted using an automatic piezoelectric head spotter before MALDI MSI analysis (Franck et al., 2009a). Specific ions from trypsin digested antibody chains were detected in the cancerous area. These experiments were performed on a MALDI LTQ Orbitrap. (A) Average mass spectrum recorded in the margin area. The arrows indicate the ions corresponding to reg alpha antibody chains after trypsin digestion and molecular MSI were performed. (B) Patient section stained with HES. The margin is indicated as a blue box. (C) Molecular images obtained by MSI of the ions at $\mathrm{m} / \mathrm{z}$ ratios of $951.64,1051.73$, $1165.76,1523.8$, and 1562.98 .

possibilities for pharmacological assays, such as monitoring the efficiency of the targeting of cytotoxic antibodies through tissue inspection. The presence and localization of therapeutic antibodies in solid tumors remain important areas of interest for investigators in this field of pharmacological development. This new method may enable the specific tracking of therapeutic or anti-marker antibodies in tissues and provide information on their presence in cancerous sections of biopsies.

A few years ago, we developed a novel technology, named Tag-Mass, which allowed for the monitoring of biomarkers in tissues. Tag-Mass involves a modified probe bearing a reporter group that is used in MALDI-MSI. The image of the marker is then indirectly generated from the probe (Lemaire et al., 2007b). This technology is compatible with all types of probes, including mRNA, antibodies, or even lectins or aptamers. It is then possible to image, with high selectivity, mRNA, antigens, oligosaccharides (including glycosylated proteins), and drugs, respectively (Gagnon et al., 2012). In the Tag-mass workflow, MALDI-MSI is combined with histological techniques, including In Situ Hybridization (ISH) and immunohistochemistry (IHC). This method allowed for the exploration of both the transcriptome and the proteome in the same tissue section by MALDI MSI in response to a single compound or a number of compounds in multiplex analyses. The latest development regarding this technology is a new UTP-tag based on click-chemistry for tracking in MALDI MSI miRNAs.

Considering this new study, SIHC and Tag-mass appear to be two complementary technologies for use in research and clinical settings. The great advantage of using the SIHC approach is its compatibility with any polyclonal or monoclonal antibodies for the detection of a given marker. Indeed, Tag Mass requires the design of a grafted antibody for the recognition of a specific antigen. Here, the approach does not require any chemical design to tag antibodies. This approach relies on polyclonal antibodies, which are known to be highly specific for a given marker of interest. In this study, we decided to focus our efforts on the C-terminal fragment of PA28, also known as Reg Alpha. This marker was extensively studied by our team and found to be present in great amounts in the cytoplasm of cancerous cells and the nucleus of normal 


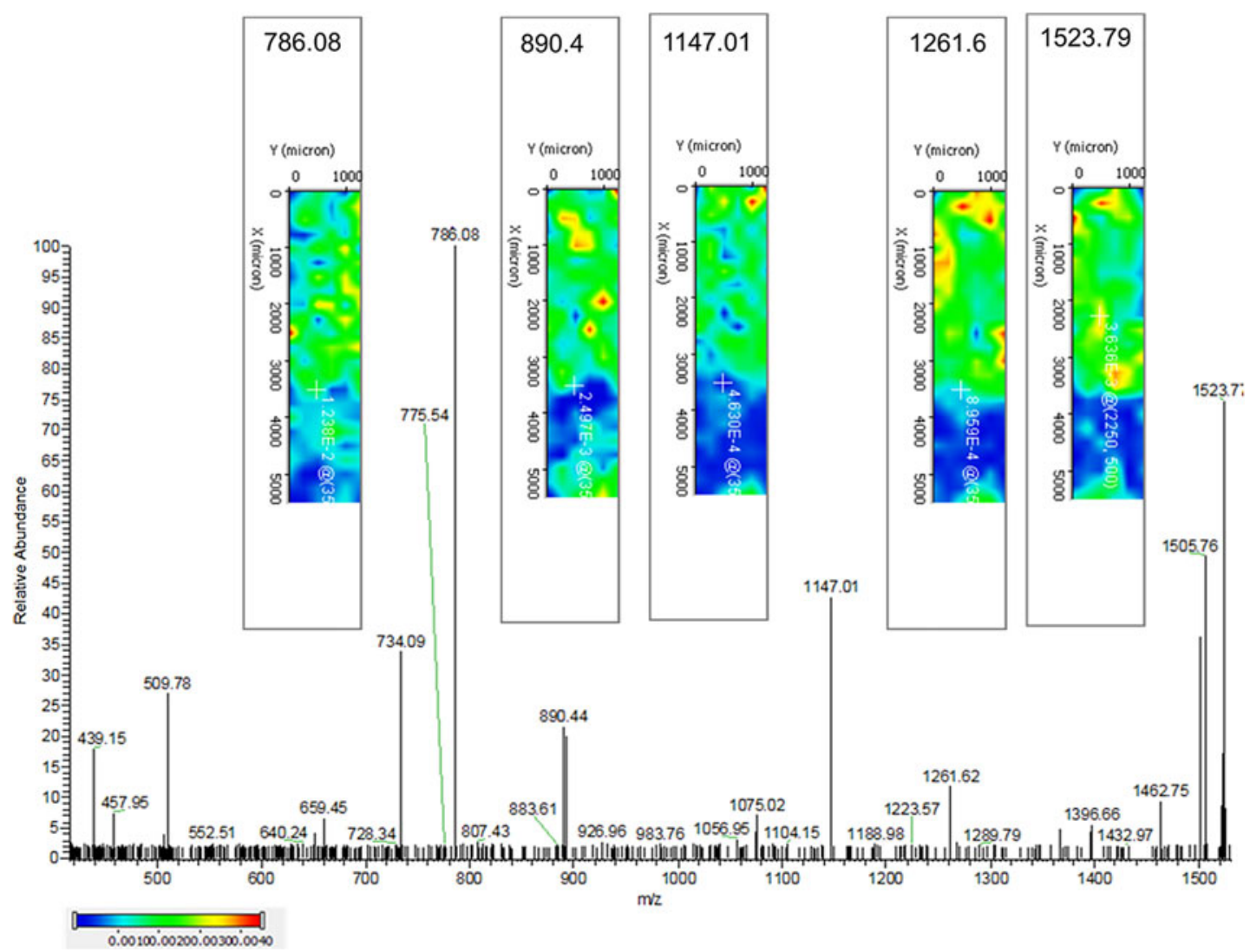

FIG. 5. MS/MS MALDI mass spectra of the ion at an $\mathrm{m} / \mathrm{z}$ value of 1523.79 detected after both tissue and in-gel digestion. Molecular images $(250 \mu \mathrm{m})$ of the parent ion and the daughter ions $(\mathrm{m} / \mathrm{z}$ ratios of 1261.6, 1147.01, 890.4, and 786.08) are presented in the inset. These experiments were performed on stage I endometrioid tissue after trypsin digestion. Trypsin and matrix (HCCA/aniline) were spotted using an automatic piezoelectric head spotter before MALDI MSI analysis (Franck et al., 2009a). Specific ions from trypsin digested antibody chains were detected in the cancerous area. These experiments were performed on a MALDI LTQ Orbitrap.

ovarian cells within the same biopsy (Lemaire et al., 2007a). We demonstrated via IHC that Cter-Reg-alpha was present in great amounts in the cancerous areas of ovarian biopsies, and this was also highlighted by mass spectrometric profiling, classical MALDI Imaging and Tag Mass MALDI Imaging (El Ayed et al., 2010). Here, we validated a strong mass spectrometric signal for this marker in the cancerous zone and a slight signal in the normal tissue area, corresponding to the recognition of the Cter part of full length PA28. In the future, we can expect increases in the imaging resolution of MALDI as new instruments are developed or improved; for example, new ionization sources coupled to highly resolutive analyzers, such as the Orbitrap (Schober et al., 2012). With this type of instrumental evolution, it may be possible to distinguish between antibody fragment signals from the nucleus in normal areas and signals from the cytoplasm in cancerous cells.

Finally, an extension of this approach is the multiplexed analysis of the markers of interest throughout the tissue section. This analysis could validate the diagnostic application of this approach by using antibodies either directed against the normal or cancerous areas of a biopsy section.

Currently, SpectroImmunoHistoChemistry will aid laboratories using mass spectrometry that aim to explore clinical applications, to cross-validate the presence of discovered an- tibodies in tissue sections and to conduct histopathological investigations on large numbers of tissue sections.

\section{Acknowledgments}

This research was supported by a collaboration between PRISM (MS) and Thermo Scientific (Bremen, Germany) and grants from the SIRIC ONCOLILLLE (to IF and MS), Ministère de L'Education Nationale, L'Enseignement Supérieur et de la Recherche, l'Agence Nationale de la Recherche (ANR PCV to IF), the ARCIR-FEDER region Nord-pas de Calais (to IF and MS), the University of Lille 1 (to IF and MS), Fonds de Recherche du Québec-Santé (FRQS to RD), CHRU of Lille (to CB), and Région Nord-Pas de Calais and Université de Sherbrooke (to RL). RD is a member of the Centre de Recherche Clinique Etienne-Le Bel (Sherbrooke, Qc, Canada).

\section{Author Disclosure Statement}

The authors declare that there are no conflicting financial interests.

\section{References}

Aichler M, Elsner M, Ludyga N, et al. (2013). Clinical response to chemotherapy in oesophageal adenocarcinoma patients is linked to defects in mitochondria. J Pathol 230, 410-419. 
Angel PM, and Caprioli RM. (2013). MALDI Imaging Mass Spectrometry: In situ Molecular Mapping. Biochemistry 52, 3818-3828.

Balluff B, Elsner M, Kowarsch A, et al. (2010). Classification of HER2/neu status in gastric cancer using a breast-cancer derived proteome classifier. J Proteome Res 9, 6317-6322.

Balluff B, Rauser S, Meding S, et al. (2011). MALDI imaging identifies prognostic seven-protein signature of novel tissue markers in intestinal-type gastric cancer. Am J Pathol 179, 2720-2729.

Bonnel D., Franck J., Meriaux C., Salzet M., and Fournier I. (2013). Ionic matrices pre-spotted MALDI plates for patients markers following, drugs titration and MALDI MSI. Anal Biochem 434, 187-198.

Bonnel D, Longuespee R, Franck J, et al. (2011). Multivariate analyses for biomarkers hunting and validation through ontissue bottom-up or in-source decay in MALDI-MSI: Application to prostate cancer. Anal Bioanal Chem 401, 149-165.

Caprioli RM, Farmer TB, and Gile J. (1997). Molecular imaging of biological samples: Localization of peptides and proteins using MALDI-TOF MS. Anal Chem 69, 4751-4760.

Chaurand P, Schwartz SA, Billheimer D, Xu BJ, Crecelius A, and Caprioli RM. (2004). Integrating histology and imaging mass spectrometry. Anal Chem 76, 1145-1155.

Cornett DS, Mobley JA, Dias EC, et al. (2006). A novel histologydirected strategy for MALDI-MS tissue profiling that improves throughput and cellular specificity in human breast cancer. Mol Cell Proteomics 5, 1975-1983.

Djidja MC, Francese S, Loadman PM, et al. (2009). Detergent addition to tryptic digests and ion mobility separation prior to MS/MS improves peptide yield and protein identification for in situ proteomic investigation of frozen and formalin-fixed paraffin-embedded adenocarcinoma tissue sections. Proteomics 9, 2750-2763.

El Ayed M, Bonnel D, Longuespee R, et al. (2010). MALDI imaging mass spectrometry in ovarian cancer for tracking, identifying, and validating biomarkers. Med Sci Monit 16, BR233-245.

Elsner M, Rauser S, Maier S, et al. (2012). MALDI imaging mass spectrometry reveals COX7A2, TAGLN2 and S100-A10 as novel prognostic markers in Barrett's adenocarcinoma. J Proteomics 75, 4693-4704.

Flatley B, Malone P, and Cramer R. (2013). MALDI mass spectrometry in prostate cancer biomarker discovery. Biochim Biophys Acta. Doi:pii: S1570—9639(13)00252-5. 10.1016/j.bbapap .2013.06.015.

Fournier I, Day R, and Salzet M. (2003). Direct analysis of neuropeptides by in situ MALDI-TOF mass spectrometry in the rat brain. Neuro Endocrinol Lett 24, 9-14.

Fournier I, Lemaire R, Wisztorski M, et al. (2006). New insight in sample preparation for MALDI imaging and new developments to approach specific MALDI imaging of the transcriptome. Proc 54rd ASMS Conf Mass Spectrometry, Seattle, Wastington, May 30-June 2.

Fournier I, Wisztorski M, and Salzet M. (2008). Tissue imaging using MALDI-MS: A new frontier of histopathology proteomics. Expert Rev Proteomics 5, 413-424.

Franck J, Arafah K, Barnes A, Wisztorski M, Salzet M, and Fournier I. (2009a). Improving tissue preparation for matrixassisted laser desorption ionization mass spectrometry imaging. Part 1: Using microspotting. Anal Chem 81, 8193-8202.

Franck J, Arafah K, Elayed M, et al. (2009b). MALDI imaging mass spectrometry: State of the art technology in clinical proteomics. Mol Cell Proteomics 8, 2023-2033.
Franck J, Longuespee R, Wisztorski M, et al. (2010). MALDI mass spectrometry imaging of proteins exceeding 30,000 daltons. Med Sci Monit 16, BR293-299.

Gagnon H, Franck J, Wisztorski M, Day R, Fournier I, and Salzet M. (2012). Targeted Mass spectrometry Imaging: Specific Targeting Mass Spectrometry imaging technologies from history to perspective. Prog Histochem Cytochem 47, 133-174.

Gustafsson JO, Eddes JS, Meding S, et al. (2012). Internal calibrants allow high accuracy peptide matching between MALDI imaging MS and LC-MS/MS. J Proteomics 75, 50935105.

Jones EA, Deininger SO, Hogendoorn PC, et al. Imaging mass spectrometry statistical analysis. J Proteomics 75, 4960-4989.

Lemaire R, Fournier I, Salzet M, et al. (2005a). Use of conjugates with linkers cleavable by photodissociation or fragmentation for mass spectrometry analysis of tissue sections. US20050687848P.

Lemaire R, Lucot J, Collinet P, et al. (2005). New developments in direct analysis by MALDI mass spectrometry for the study of ovarian cancer. Mol Cell Proteomics 4, S306-S306.

Lemaire R, Lucot JP, Collinet P, Vinatier D, Tabet JC, Salzet M, and Fournier I. (2005b). New developments in direct analyses by MALDI mass spectrometry for study ovarian cancer. Mol Cell Proteomics 4, S305-S308.

Lemaire R, Menguellet SA, Stauber J, et al. (2007a). Specific MALDI imaging and profiling for biomarker hunting and validation: Fragment of the $11 \mathrm{~S}$ proteasome activator complex, Reg alpha fragment, is a new potential ovary cancer biomarker. J Proteome Res 6, 4127-4134.

Lemaire R, Stauber J, Wisztorski M, et al. (2007b). Tag-mass: Specific molecular imaging of transcriptome and proteome by mass spectrometry based on photocleavable tag. J Proteome Res 6, 2057-2067.

Lemaire R, Wisztorski M, Desmons A, et al. (2006). MALDI-MS direct tissue analysis of proteins: Improving signal sensitivity using organic treatments. Anal Chem 78, 7145-7153.

Longuespee R, Boyon C, Castellier C, et al. (2012a). The C-terminal fragment of the immunoproteasome PA28S (Reg alpha) as an early diagnosis and tumor-relapse biomarker: Evidence from mass spectrometry profiling. Histochem Cell Biol 138, $141-154$

Longuespee R, Boyon C, Desmons A, et al. (2012b). Ovarian cancer molecular pathology. Cancer Metastasis Rev 31, 713732.

Longuespee R, Gagnon H, Boyon C, et al. (2013). Proteomic analyses of serous and endometrioid epithelial ovarian cancers-Cases studies-Molecular insights of a possible histological etiology of serous ovarian cancer. Proteomics Clin Appl 7, 337-354.

Longuespée RB, Kerdraon O, Vinatier D, Fournier I, Day R, and Salzet M. (2012). MALDI MSI and ovarian cancer biomarkers. Adv Cancer Manag 211-236.

Lowe KA, Chia VM, Taylor A, et al. (2013). An international assessment of ovarian cancer incidence and mortality. Gynecol Oncol 130, 107-114.

McCombie G, Staab D, Stoeckli M, and Knochenmuss R. (2005). Spatial and spectral correlations in MALDI mass spectrometry images by clustering and multivariate analysis. Anal Chem 77, 6118-6124.

McDonnell LA, Corthals GL, Willems SM, van Remoortere A, van Zeijl RJ, and Deelder AM. (2010). Peptide and protein imaging mass spectrometry in cancer research. J Proteomics 73, 1921-1944. 
Meding S, Balluff B, Elsner M, et al. (2012a). Tissue-based proteomics reveals FXYD3, S100A11 and GSTM3 as novel markers for regional lymph node metastasis in colon cancer. J Pathol. Doi: 10.1002/path.4021.

Meding S, Nitsche U, Balluff B, et al. (2012b). Tumor classification of six common cancer types based on proteomic profiling by MALDI imaging. J Proteome Res 11, 1996-2003.

Meding S, and Walch A. (2013). MALDI imaging mass spectrometry for direct tissue analysis. Methods Mol Biol 931, 537546.

Ray-Coquard I, Chauvin F, Leblanc E, et al. (2012). [PAIR-gynaecology: M-4ulti/interdisciplinary for gynecologic cancer research. Problems needed to be resolved]. Bull Cancer 99, 479-98.

Schober Y, Guenther S, Spengler B, and Rompp A. (2012). Highresolution matrix-assisted laser desorption/ionization imaging of tryptic peptides from tissue. Rapid Commun Mass Spectrom 26, 1141-1146.

Schwamborn K, Krieg RC, Reska M, Jakse G, Knuechel R, and Wellmann A. (2007). Identifying prostate carcinoma by MALDI-Imaging. Int J Mol Med 20, 155-159.

Seeley EH, and Caprioli RM. (2008). Imaging mass spectrometry: Towards clinical diagnostics. Proteomics Clin Appl 2, 14351443.

Stauber J, El Ayed M, Wisztorski M, Day R, Fournier I, and Salzet M. (2009). Polymerase chain reaction and immunoassay-matrix assisted laser desorption mass spectrometry using tag-mass technology: New tools to break down quantification limits and multiplexes. Anal Chem 81, 9512-9521.

Stauber J, Lemaire R, Franck J, et al. (2008). MALDI imaging of formalin-fixed paraffin-embedded tissues: Application to model animals of Parkinson disease for biomarker hunting. J Proteome Res 7, 969-978.

Stauber J, Lemaire R, Wisztorski M, et al. (2006). New developments in MALDI imaging mass spectrometry for pathological proteomic studies: Introduction to a novel concept, the specific MALDI imaging. Mol Cell Proteomics 5, S247-S249.

Trede D, Kobarg JH, Oetjen J, Thiele H, Maass P, and Alexandrov T. (2012). On the importance of mathematical methods for analysis of MALDI-imaging mass spectrometry data. J Integr Bioinform 9, 189.

Wisztorski M, Fatou B, Franck J, et al. (2013). Microproteomics by liquid extraction surface analysis: Application to FFPE tissue to study the fimbria region of tubo-ovarian cancer. Proteomics Clin Appl 7, 234-240.

Address correspondence to: Dr. Isabelle Fournier

or

Dr. Michel Salzet Laboratoire PRISM: Protéomique, Réponse Inflammatoire, Spectrométrie de Masse, EA 4550

Université de Lille 1

Cité Scientifique 59650 Villeneuve D'Ascq

Lille Cedex

France

E-mail: isabelle.fournier@univ-lille1.fr

or

Michel.salzet@univ-lille1.fr 